\title{
Laparoscopic Management of Adhesive Small Bowel Obstruction
}

\author{
Ferid Konjic ${ }^{1}$, Enes Idrizovic ${ }^{1}$, \\ Ismar Hasukic ${ }^{2}$, Alen Jahic ${ }^{3}$ \\ ${ }^{1}$ Clinic for Surgery, University Clinical \\ Center Tuzla, Tuzla, Bosnia and \\ Herzegovina \\ ${ }^{2}$ Clinic for Internal disease, University \\ Clinical Center Tuzla, Tuzla, Bosnia \\ and Herzegovina \\ ${ }^{3}$ Clinic for Cardiovascular disease, \\ University Clinical Center Tuzla, Tuzla, \\ Bosnia and Herzegovina
}

Corresponding author: Ferid Konjic, MD. Clinic for Surgery, University Clinical Center Tuzla, Tuzla, Bosnia and Herzegovina. Tel: +38761 179 118. E-mail:

\section{doi: 10.5455/aim.2016.24.69-71}

ACTA INFORM MED. 2016 FEB; 24(1): 69-71 Received: 11 October 2015 - Accepted: 15 December 2015

\begin{abstract}
Introduction: Adhesions are the reason for bowel obstruction in $80 \%$ of the cases. In well selected patients the adhesive ileus laparoscopic treatment has multiple advantages which include the shorter hospitalization period, earlier food taking, and less postoperative morbidity rate. Case report: Here we have a patient in the age of 35 hospitalized at the clinic due to occlusive symptoms. Two years before an opened appendectomy had been performed on him. He underwent the treatment of exploration laparoscopy and laparoscopic adhesiolysis. Dilated small bowel loops connected with the anterior abdominal wall in the ileocecal region by adhesions were found intraoperatively and then resected harmonically with scalpel. One strangulation around which a small bowel loop was wrapped around was found and dissected. Postoperative course was normal.
\end{abstract}

Key words: adhesive ileus, laparoscopy.

\section{INTRODUCTION}

Adhesions are the reason for bowel obstruction in $80 \%$ of the cases. Less common reasons are: peritoneal carcinomatosis, Crohn's disease, internal hernia, diaphragmatic hernia, Meckel's diverticulum, and billiard ileus (1). Due to objective reasons, laparoscopy has no widespread application at small bowel obstruction yet. If applicable, the diagnostic laparoscopy in smaller adhesions can be transformed in therapeutic one (2). The first laparoscopic adhesiolysis in small bowel obstruction was performed by Mouret in 1972. The first following case, the use of laparoscopy in small bowel obstruction was accepted by other surgeons and the indication was in patients with one adhesion and with no clinical signs of ischemia and small bowel necrosis (3). At small bowel laparoscopic adhesiolysis we have to stick to some valid principles. Firstly the operational technique is specific: the first trocar has to be placed by using the Hasson's technique for open laparoscopy in order to avoid accidental perforations of intestines which are distended and adhered to the anterior abdominal wall. Two $5 \mathrm{~mm}$ trocars must be inserted under direct vision in order to explore the peritoneal cavity.
A surgeon performs laparotomy if necrotic and ischemic intestines are registered, and contrary if healthy intestines are registered the laparoscopic procedure can be done, with the atraumatic pincer to isolate the adhesion, coagulating the adhesion with bipolar coagulation and then cuts it with scissors. There are three key factors during the procedure of laparoscopic adhesiolysis: early indication for surgical treatment, the exclusion of patients with the doubt to small bowel strangulation or torsion accompanied with ischemia and small bowel necrosis (4). In our work, we have presented a patient with adhesive postoperative small bowel ileus where the adhesiolysis had been successfully performed by laparoscopy.

\section{CASE REPORT}

Male, 35 years of age, admitted to a surgery clinic for abdominal pains localized in periumbilical region, a type of colic, followed by vomiting and constipation in the last 24 hours. The patient stated that he had undergone a prior appendix surgery performed by a classic technique two years before. Physical examination confirmed that the patient was hemodynamically and respiratory stable, afebrile $\mathrm{T}=37^{\circ} \mathrm{C}$; 


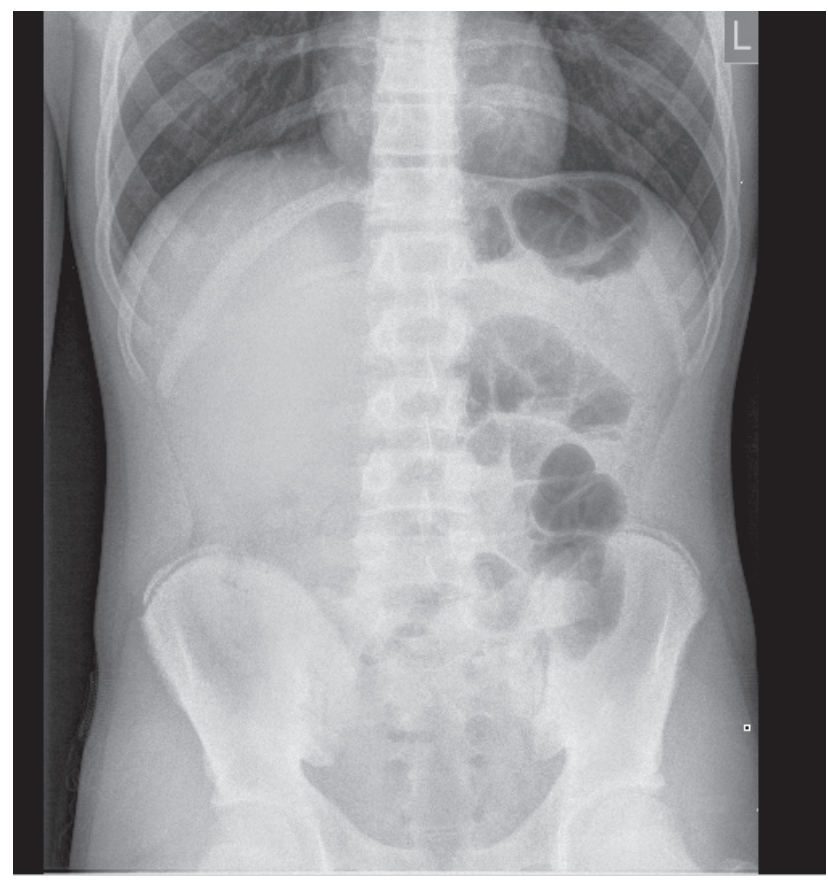

Figure 1. Native abdominal RTG: air filled and moderately distended small bowel loops visible in the projection of the left hemiabdomen.

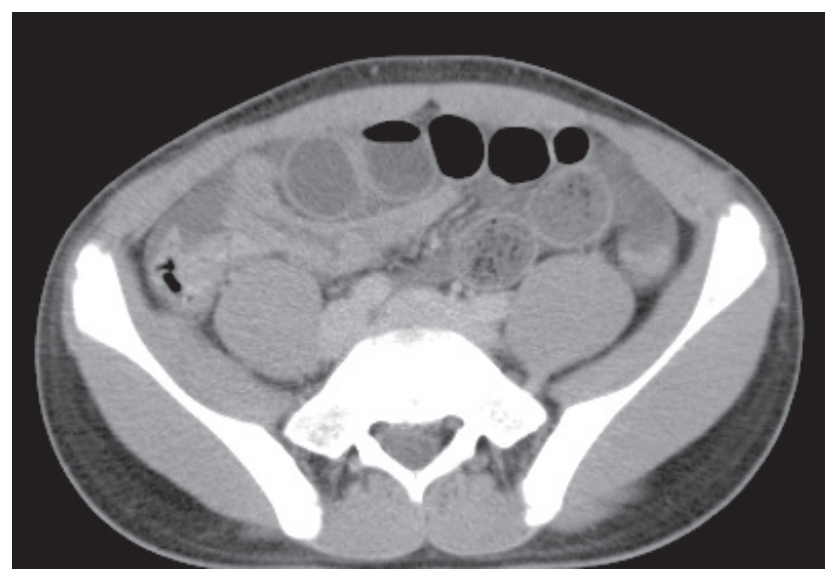

Figure 2. Abdominal CT with contrast

blood pressure 120/80 $\mathrm{mmHg}$; pulse 75 beats per minute; cardiovascular and respiratory findings normal; $3 \mathrm{~cm}$ long postoperative cut in the abdomen parallel to thorax in the right lower quadrant, palpatory painfully sensitive in periumbilical region. The urologist excluding the acute urological disease was consulted. Abdomen ultrasound shows dilated small bowel loops and free liquid in perisplenic area and Pouch of Douglas. Laboratory findings show higher Le values in blood $15.40 \mathrm{~g} / \mathrm{l}$, differentiated blood count shows 83.8\% neutrophilia, CRP value 1.40. Native abdomen RTG shows air filled and moderately distended small bowel loops (Figure 1). Abdominal CT was performed with the contrast showing peri and subhepathic, perisphlenic, interintestinal, Aeffusion with the propagation in pelvis, limpid fluid density and moderately distended ileum loops, diameter up to $33 \mathrm{~mm}$, full of liquid, with the thicken wall in the upper pelvic cavity and narrowed lumen. (Figure 2). In the first three days of hospitalization the patient shows improvement with the applied conservative treatment. On the fourth day, the patient complaints that he has pains in the abdomen again. The pains are followed with fibrillation $38.2^{\circ} \mathrm{C}$. The gastroenterologist was consulted and he performed the colonoscopy, $\mathrm{X}$ rayscopia of digestive tube and serum ascit albumin gradient. The colonoscopy findings were normal. For all the above mentioned the decision was to perform exploration laparoscopy. The access into the abdomen was Open Hasson's Technique. Dilated, spastic small bowel loops connected with the anterior abdominal wall in the ileocecal region by adhesions were found intraoperatively and then resected harmonically with scalpel after which one strangulation around which a small bowel loop was wrapped around was found and dissected. The surgery finished by placing the drain into Pouch of Douglasm (Figure 3 and 4). Postoperative course passed normally. Due to the diarrhea on the third day the infectologist had been consulted and the appropriate therapy was prescribed. Completely recovered, with normal general and local findings, the patient was discharged to home treatment on the sixth postoperative day.

\section{DISCUSSION}

Good surgery technique, open Hasson's laparoscopic access and the possibility of moving the operating table in different positions are needed to perform laparoscopic adhesiolysis (5). The causes of laparotomic conversion are the aggravated exposition and fibrous adhesion treatment, then the reduced operational field caused by the small bowel dilatation, multiple adhesions and sometimes with the presence of posterior peritoneal fibrous adhesion that are treated harder than laparoscopically (6). Other causes of conversion are the presence of intestines necrosis, which always demands the resection which is primarily performed laparotomically. One of the causes of conversion is inadvertent enterotomy. Suturation which is performed by open access gives more certainty and durability, especially when performed on dilated and fragile intestine. In order to avoid accidental enterotomies, several strategies have been developed: exclusion of patients with a history of multiple abdominal surgeries, good surgical technique which implies dissection close to parietal peritoneum, avoid cutting the tenacious fibrous adhesions and always control the direction of the instruments (7).

The predictive factors for successful laparoscopic adhesiolysis are non-medial laparotomy (Mc Burney incision), less

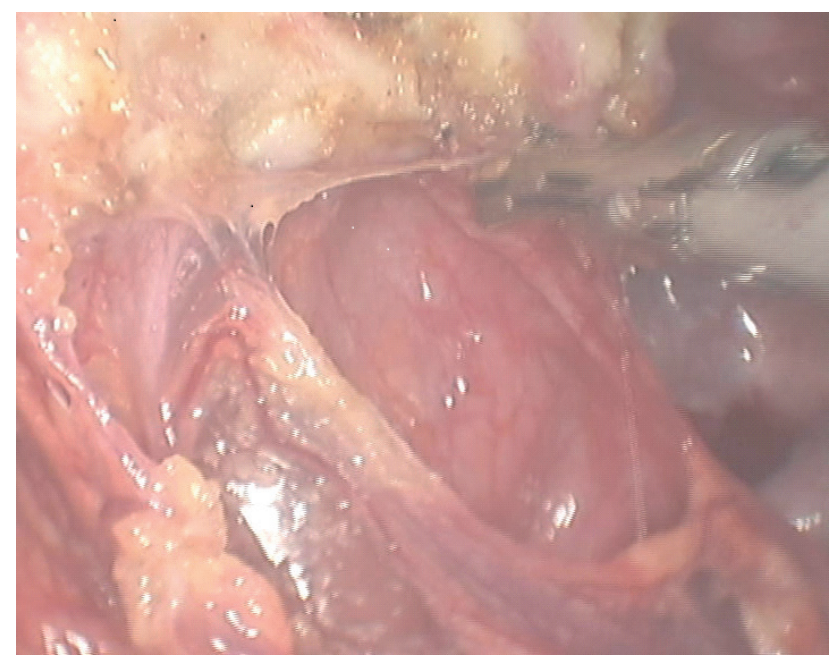

Figure 3. Laparoscopic adhesiolysis between the small bowel loops and anterior abdominal wall. 


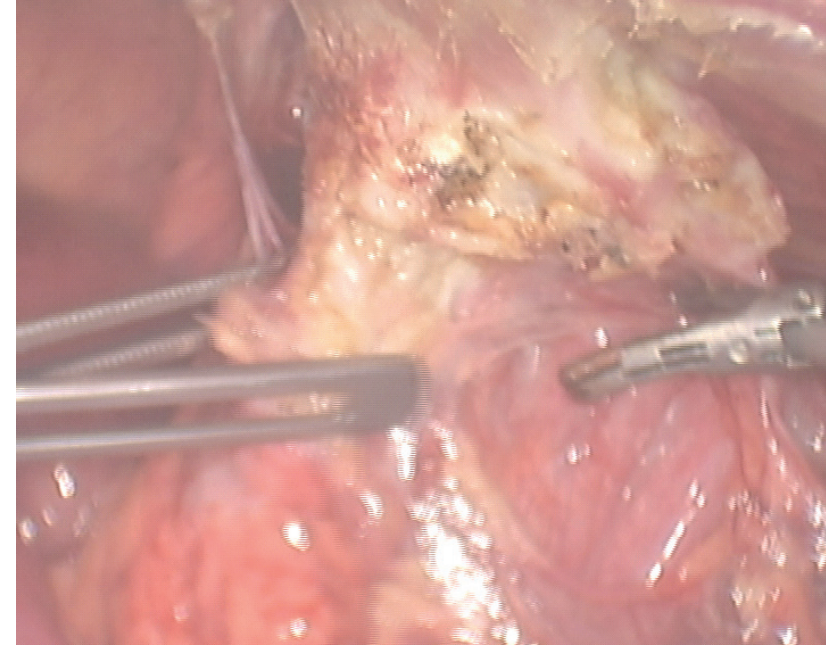

Figure 4. Laparoscopic resection of strangulation around which the small bowel loop had adhered.

than two laparotomies, one fibrous adhesion causing obstruction, lack of peritoneal stimulus during physical examination (performing the surgery within 24 hours from the occurrence of symptoms (8). On the other side, number of laparotomies more than two, the presence of multiple adhesions can be considered a relative contraindication (9). Absolute contraindications are the presence of intestines necrosis due to the obstruction, enormously dilated loops because they narrow the operative field, serious comorbidity such as cardiovascular, respiratory and hemostatic disorders (10). Through the comparison of laparoscopic and laparotomic access at small bowel obstruction the following was determined: the duration of surgery is longer at laparoscopy compared to open surgery (11). The duration of hospitalization, initiation of peristalsis, postoperative morbidity rate is lower with laparoscopic approach (12). With laparoscopic adhesiolysis we avoid laparotomy, which can cause another adhesion and bowel obstruction (8).

\section{CONCLUSION}

Laparoscopic adhesiolysis at small bowel obstruction is feasible but suitable only if performed by an experienced laparoscopic surgeon with selected patients. The appropriate selection of patients is essential in order to avoid the increased morbidity rate noticed due to laparatomic conversion. The factors to be considered are: the number of previous laparatomies, a type of surgical procedure, adhesion degree, the time elapsed from the beginning of symptoms, the degree of bowel loops dilatation in X-ray images, conjunction with ischemia and intestines necrosis. With the presentation of laparoscopic adhesiolysis of our patient we want to show that this technique is safe and reliable even in our conditions.

Author's contribution: author and all co-authors of this paper have contributed in all phases if it's preparing. Final proof reading was made by first author.

Conflict of interest: none declared.

\section{REFERENCES}

1. Gutt CN, Oniu T, Schemmer P, Mehrabi A, Buchler MW. Fewer adhesions induced by laparoscopic surgery? Surg Endosc. 2004; 18: 1202-7.

2. Zerey M, Sechrist CW, Kercher KW, Sing RF, Matthews BD, Heniford BT. Laparoscopic management of adhesive small bowel obstruction. Am Surg. 2007; 73: 773-8.

3. Mouret P. L'adesiolisi coelioscopia. In: Testas P, Delaitre B, editor. Chirurgia digestiva per via coelioscopica. Edizioni Vigot, Friburgo; 1994: 53-69.

4. Farinella E, Cirocchi R, La Mura F, Morelli U, Cattorini L, Delmonaco P, Migliaccio C, A De Sol A, Cozzaglio L, Sciannameo F. Feasibility of laparoscopy for small bowel obstruction. World J Emerg Surg. 2009; 19(4): 3.

5. Strickland P, Lourie DJ, Suddleson EA, Blitz JB, Stain SC. Is laparoscopic safe and effective for treatment of acute small-bowell obstruction? Surg Endosc. 1999; 13: 695-8.

6. Parent S, Tortuyaux JM, Deneuvile M. What are the small bowel obstruction to operate and how to do it? Acta Gastroentrol Clin Biol. 1996; 20: 357-61.

7. Camazine B. The medicolegal fallout from laparoscopic bowel injury. Cont Surg. 2004; 60: 380-1.

8. Perniceni T. Traitement laparoscopique des occlusions aigues de l'intestin grele: limites et indications. Referentiel Association Francaise de Chirurgie (AFC) nº4513 créé(e) le 28/04/05 par Pr Denis Collet Prevention et traitement des occlusions du grele su bride.

9. Benoist S, De Wateville JC, Gayral F. Place de la coelioscopie dans les occlusions aigues du grele. Gastroenterol Clin Biol. 1996; 20: 357-61.

10. Szomstein S, Lo Menzo E, Simpfendorfer C, Zundel N, Rosenthal R. Laparoscopic lysis of adhesions. World J Surg. 2006; 30: 535-40.

11. Wullstein C, Gross E. Laparoscopic compared with conventional treatment of acute adhesive small bowel obstruction. $\mathrm{Br} \mathrm{J}$ Surg. 2003; 90: 1147-51.

12. Khaikin M, Schneidereit N, Cera S, Sands D, Efron J, Weiss G, Nogueras JJ, Vernava AM, Wexner SD. Laparoscopic vs. open surgery for acute adhesive small-bowel obstruction: patient' outcome and cost-effextiveness. Surg Endosc. 2007; 21: 742-6. 